\title{
Cascaded Multifunctional Metasurfaces for Single-shot Quantitative Phase Gradient Microscopy
}

\author{
Hyounghan Kwon, Ehsan Arbabi, Seyedeh Mahsa Kamali, MohammadSadegh Faraji-Dana, Andrei Faraon* \\ T. J. Watson Laboratory of Applied Physics, California Institute of Technology, 1200 E. California Blvd., Pasadena, CA 91125, USA \\ Department of Electrical Engineering, California Institute of Technology, 1200 E. California Blvd, Pasadena, CA, USA \\ *faraon@caltech.edu
}

\begin{abstract}
We experimentally demonstrate a single-shot quantitative phase gradient microscope by two cascaded multifunctional metasurfaces. Experiments with various specimens show the capability to capture quantitative phase gradient images with single-cell resolution and low noise levels. (C) 2020 The Author(s)

OCIS codes: (050.6624) Subwavelength structures; (110.6880) Three-dimensional image acquisition
\end{abstract}

Optical phase imaging techniques capture images of transparent samples such as biological cells. For the unlabeled cells, phase information represents their morphological properties. The phase imaging techniques also enable long-term investigation of living cells by avoiding photobleaching commonly faced in fluorescence imaging. Quantitative phase imaging (QPI) has been rapidly developed in the past two decades, as it has overcome limits of conventional qualitative phase microscopy such as phase contrast and differential interference contrast (DIC) microscopy [1]. Thus, QPI is considered as a complementary imaging method to the fluorescence imaging. However, QPI systems usually require interferometry setups. This hinders the development of the miniaturized QPI microscopes which can be of interests in various fields such as biomedicine and point-of-care.

In a decade, dielectric metasurfaces have been considered as alternatives of conventional refractive or diffractive optical elements because they provide unprecedented optical properties such as multifunctionality with a compact footprint and high efficiency [2]. The dielectric metasurfaces have enabled various optical devices such as compact spectrometers and endoscopes [3,4]. In addition, integration of multiple metasurfaces further enhanced their functionalities [5]. Despite rapid advances of the metasurfaces, potentials of the metasurfaces for QPI have not been explored. Here, we propose a concept of a single-shot quantitative phase gradient microscope (QPGM) consisting of two vertically cascaded multifunctional metasurfaces layers [6]. We experimentally demonstrate millimeter-scale optical devices capturing a phase gradient image (PGI) from various phase samples.

Figure 1a conceptually illustrates the idea of the QPGM composed of two metasurface layers. The metasurface layer 1 captures two slightly shifted images for TE and TM polarizations. It also splits the captured images into three separate directions towards three off-axis birefringent lenses in the metasurface layer 2 . Thus, the metasurface layer 2 effectively constitutes three DIC microscopes with the metasurface layer 1. Polarization and spatial multiplexing techniques are employed in the design of both metasurface layers [7,8]. For a binary phase target in Fig. 1b, the QPGM captures three DIC images shown in Fig. 1c. A PGI shown in Fig. 1d can be calculated from the images in Fig. 1c. To implement the concept of the QPGM, we design and fabricate the metasurface layers consisting of the amorphous silicon nano-posts on two separate fused silica substrates. Optical images and a scanning electron microscope image of the fabricated metasurfaces are shown in Fig. 1f.

We experimentally verify the QPGM's capability of capturing PGIs of various phase samples. With a CMOS image sensor, the two metasurface layers constitute a compact optical setup shown in Fig. 1g. First, a 1951 USAF phase resolution target is used as a test target and Figure 1h shows the PGI of the target captured by the setup in Fig. 1g. Sea urchin samples are also imaged in Fig. 1i. The results show the QPGM's capability of capturing phase gradient information form biological samples. To more rigorously test the quantitative imaging performance, the thickness achieved with the QPGM is compared with the thickness measured by AFM with the phase resolution targets having seven different thicknesses. The estimated thicknesses by the QPGM are averaged over 100 arbitrarily chosen points on the sample edges. The estimated thicknesses are plotted in Fig. 1i and show good agreement with the values measured by AFM. In addition, the measured lateral resolutions along the $x$ and $y$ axes are $2.76 \mu \mathrm{m}$ and $3.48 \mu \mathrm{m}$, respectively. Moreover, the measured temporal and spatial noise levels are as small as 11.4 and $36.9 \pm 0.7 \mathrm{rad} \mu \mathrm{m}-1$, respectively. For further miniaturization, two metasurface layers are additionally designed and fabricated on a single fused silica substrate. Figure $1 \mathrm{j}$ shows a schematic of the double-sided QPGM. The PGI measured by the double-sided QPGM is shown in Fig $1 \mathrm{k}$. 
In conclusion, we utilized the two multifunctional metasurface layers to realize the miniaturized QPGMs. This work demonstrates metasurfaces' potentials in QPI systems. With the current great interests in QPI and rapid evolution of the metasurfaces, we envision that the metasurfaces will play a significant role in the development of QPI.

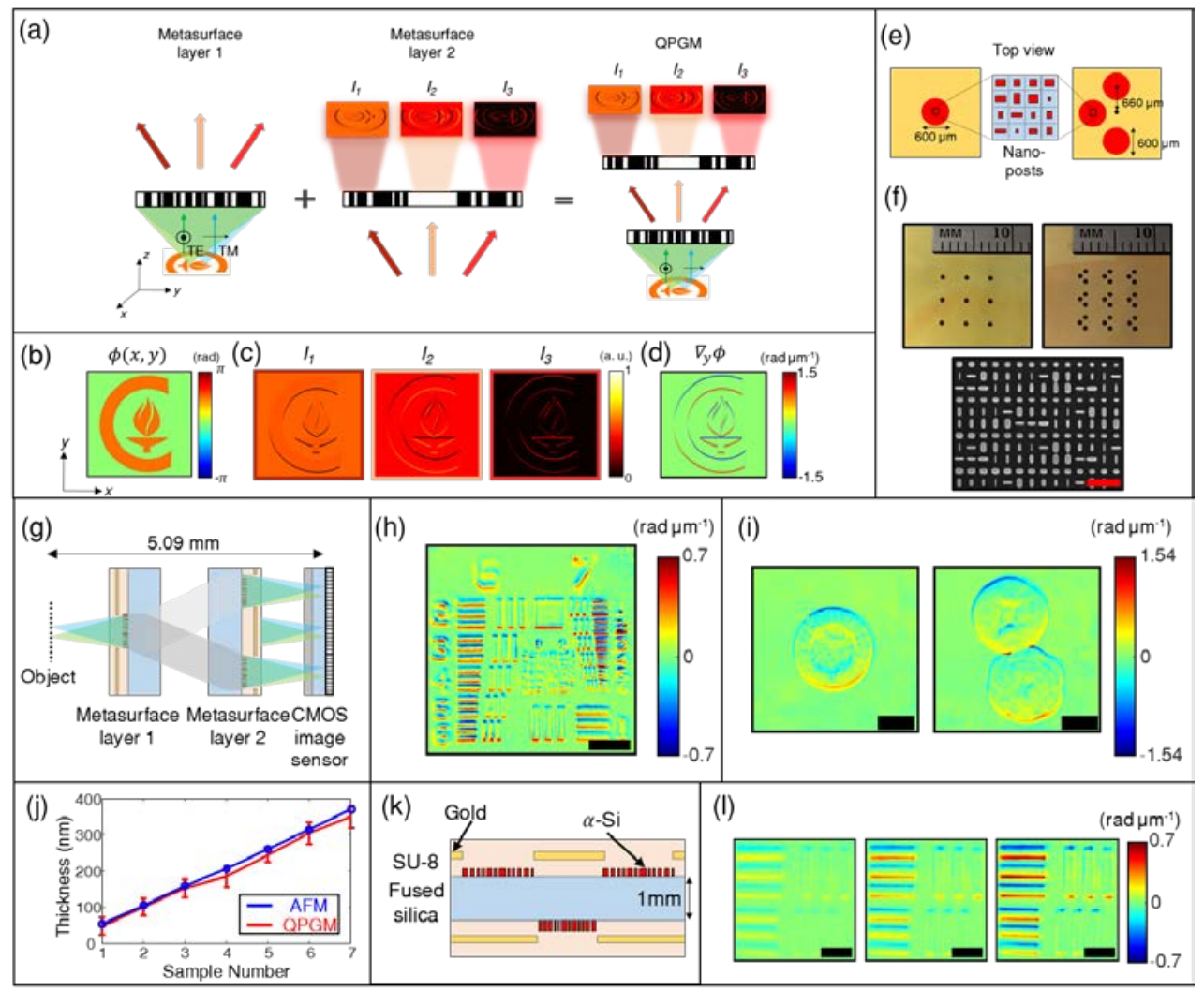

Fig. 1. a, Schematic illustration of QPGM. Roles of the two metasurface layers are illustrated. b, An example phase target with unity amplitude. c, Set of three DIC images and d, PGI of the phase sample shown in b. e, Schematic top views of the metasurface layers 1 (left) and 2 (right). f, Optical images (top) and a scanning electron microscopy image (bottom) of fabricated metasurfaces. Scale bar is $1 \mu \mathrm{m}$. $\mathbf{g}$, Schematic of the miniaturized optical set-up. h, PGI of a resolution target captured by the setup in e. Scale bar is $50 \mu \mathrm{m}$. i, PGIs of sea urchin samples. Scale bars are $40 \mu \mathrm{m}$. j. Thicknesses of seven different phase targets estimated by the QPGM (red), and those measured by AFM (blue). Error bars represent standard deviations of the estimated values. k..Schematic side view of the double-sided QPGM. I, PGIs captured for three parts of the resolution targets with thicknesses of $105 \mathrm{~nm}, 207 \mathrm{~nm}$ and $314 \mathrm{~nm}$ (left to right), respectively. Scale bars are $15 \mu \mathrm{m}$.

[1] Y. Park, C. Depeursinge, and G. Popescu, “Quantitative phase imaging in biomedicine,” Nat. Photonics 12, 578-589 (2018).

[2] S. M. Kamali, E. Arbabi, A. Arbabi, and A. Faraon, "A review of dielectric optical metasurfaces for wavefront control," Nanophotonics 7(6), 1041-1068 (2018).

[3] M. Faraji-Dana, E. Arbabi, A. Arbabi, S. M. Kamali, H. Kwon, and A. Faraon, "Compact folded metasurface spectrometer,” Nat. Commun. 9, 4196(2018).

[4] H. Pahlevaninezhad, M. Khorasaninejad, Y.-W. Huang, Z. Shi, L. P. Hariri, D. C. Adams, V. Ding, A. Zhu, C.-W. Qiu, F. Capasso, and M. J. Suter, "Nano-optic endoscope for high-resolution optical coherence tomography in vivo," Nat. Photonics 12(9), 540-547 (2018).

[5] A. Arbabi, E. Arbabi, S. M. Kamali, Y. Horie, S. Han, and A. Faraon, "Miniature optical planar camera based on a wide-angle metasurface doublet corrected for monochromatic aberrations," Nat. Commun. 7, 13682 (2016).

[6] H. Kwon, E. Arbabi, S. M. Kamali, M. Faraji-Dana and A. Faraon, "Single-shot quantitative phase gradient microscopy using a system of multifunctional metasurfaces," Nat. Photonics (2019).

[7] A. Arbabi, Y. Horie, M. Bagheri, and A. Faraon, "Dielectric metasurfaces for complete control of phase and polarization with subwavelength spatial resolution and high transmission," Nat. Nanotechnol. 10, 937-943 (2015).

[8] E. Maguid, I. Yulevich, D. Veksler, V. Kleiner, M. L. Brongersma, and E. Hasman, "Photonic spin-controlled multifunctional shared-aperture antenn-a array," Science 352(6290), 1202-1206 (2016). 